Check for updates

Cite this: RSC Adv., 2017, 7, 44097

\title{
Magnetic properties of ordered polycrystalline FeRh thin films
}

Jingfan Ye, ${ }^{a}$ Marco Hauke, ${ }^{a}$ Vikram Singh, ${ }^{b}$ Rajeev Rawat, ${ }^{\text {b }}$ Mukul Gupta, ${ }^{\mathrm{b}}$ Akhil Tayal, ${ }^{\mathrm{c}}$ S. M. Amir, Jochen Stahn ${ }^{\mathrm{e}}$ and Amitesh Paul (D) *a

FeRh alloys with B2-order have been investigated earlier in epitaxial films as the metamagnetic transition temperature $\left(T_{\mathrm{AF}-\mathrm{FM}}\right)$ depends upon the structure and lattice strain or disorder. Here, we report on an ordered polycrystalline FeRh film on $\mathrm{Al}_{2} \mathrm{O}_{3}$ (0001) deposited by $\mathrm{DC}$ magnetron sputtering. We found that $T_{\mathrm{AF}-\mathrm{FM}}=346 \mathrm{~K}$ (in $1 \mathrm{~T}$ ), which can be shifted by a magnetic field to $288 \mathrm{~K}$ (in $7 \mathrm{~T}$ ) with similar rates (dT/ $\mathrm{d} H$ ) of $-8.5 \mathrm{~K} \mathrm{~T}^{-1}$ (heating) and $-9.6 \mathrm{~K} \mathrm{~T}^{-1}$ (cooling) as in epitaxial ones. However, a significant difference in the rate of domain nucleation and propagation with temperature $(d M / d T)$ during cooling and heating procedures is noted. These changes can be ascribed to ordered and disordered polycrystallinity of the film. Furthermore, polarized neutron reflectometry (PNR) shows that the FeRh film is not homogeneous throughout the depth of the film. On the one hand, we found evidence of $\mathrm{Fe}$ segregation at the surface of the film extended to within a few nanometers from the top-interface and on the other hand, Rh richness at the substrate-interface. A gradient in Fe at\% has resulted in changing magnetic phases from ordered bcc (B2) within the film to a disordered bcc (A2) structure at the surface and a disordered fcc phase (A1) at the bottom. Thus it is possible to grow commercially viable polycrystalline films with similar characteristics to those of epitaxial films.

Received 16th June 2017

Accepted 28th August 2017

DOI: $10.1039 / \mathrm{c} 7 \mathrm{ra06738k}$

rsc.li/rsc-advances moment well below the $T_{\mathrm{AF}-\mathrm{FM}}$ which is confined to a few nanometers near the interfaces due to strain at the substrate and Rh enrichment at the top. ${ }^{6}$ In FeRh/BTO thin film heterostructures, it was shown that the strain and field effects in ferroelectric $\mathrm{BaTiO}_{3}$ (BTO) can shift the metamagnetic transition temperature of FeRh to just above room temperature. ${ }^{7}$ Very recently, lattice strain has been shown to reduce $T_{\mathrm{AF}-\mathrm{FM}}$ to $200{ }^{\circ} \mathrm{C}$ in disordered FeRh films. ${ }^{8}$ The occurrence of martensitic lattice instabilities in $\mathrm{Fe}-\mathrm{Rh}$ have been reported in B2-ordered 50-50 alloys induced by deformation., ${ }^{\mathbf{9} 10}$ The structural and compositional disorder is known to strongly influence the magnetic properties. Thereby, such effects of disorder can be explored in ordered and disordered polycrystalline films as well.

Here, we have deposited an ordered polycrystalline FeRh film on $\mathrm{Al}_{2} \mathrm{O}_{3}$ (0001) by magnetron sputtering. We found that $T_{\mathrm{FM}-\mathrm{AF}}=346 \mathrm{~K}(1 \mathrm{~T})$ and could be shifted by an applied magnetic field to $288 \mathrm{~K}(7 \mathrm{~T})$ with similar $\mathrm{d} T / \mathrm{d} H$ rates of around $-8.5 \mathrm{~K} \mathrm{~T}^{-1}$ (heating) and $-9.6 \mathrm{~K} \mathrm{~T}^{-1}$ (cooling) as in epitaxial ones reported in the literature. The significant difference in the rate of domain nucleation and propagation with temperature $(\mathrm{d} M / \mathrm{d} T)$ during cooling and heating procedures is noted and can be ascribed to the polycrystallinity of the film. Polarized neutron reflectometry (PNR) shows that the FeRh film is not homogeneous throughout the depth of the film as we found evidence of Fe segregation at the top-interface of the film and $\mathrm{Rh}$ enrichment at the substrate-interface. The Fe segregated 
portion has a lower magnetization than the film due to a disordered bcc phase, while the bottom is enriched with Rh.

\section{Methods}

\section{Sample preparation}

One may note that a slight imbalance in the compositional percentage destroys or affects the metamagnetic properties drastically. We have tried extensively to optimize the $50-50 \%$ $\mathrm{Fe}-\mathrm{Rh}$ with various estimated compositions. The reproducibility of the films is not dependent so much upon the thickness, rather on the deposition parameters. The parameters were optimized after rigorous due diligence.

A nominal equiatomic FeRh thin film was deposited on an $\mathrm{Al}_{2} \mathrm{O}_{3}$ (0001) substrate by DC magnetron sputtering (AJA ORION8). Pure Fe (99.995\%) and Rh (99.9\%) targets were co-sputtered at a substrate temperature of $700{ }^{\circ} \mathrm{C}$. The base pressure of the preparation chamber was $2 \times 10^{-7}$ Torr and during deposition the pressure was 2 mTorr due to a flow of Ar gas at $10 \mathrm{sccm}$. Before deposition, the substrates were cleaned at $500{ }^{\circ} \mathrm{C}$ using an rf bias plasma at a power of $100 \mathrm{~W}$ for 15 minutes. The targets were pre-sputtered to remove a native oxide layer. The substrates were rotated at a constant speed of $60 \mathrm{rpm}$ for better thickness and composition homogeneity. After deposition the films were annealed at $700{ }^{\circ} \mathrm{C}$ for 30 minutes.

\section{X-ray and secondary ion mass spectroscopy (SIMS) characterization}

X-ray reflectivity (XRR) and diffraction (XRD) measurements were carried out at the $\mathrm{Cu}-\mathrm{K} \alpha$ wavelength on an Empyrean diffractometer from PANalytical, providing information on the layer structure and crystallinity of the layer.

The secondary ion mass spectroscopy (SIMS) measurements were performed with O-secondary ions with a kinetic energy of $15 \mathrm{keV}$, while positive (ionized) secondary ions were detected on the detector.

\section{Magnetometry}

Conventional in-plane magnetization loops were measured at various temperatures and fields using a superconducting quantum interference device (SQUID) from Quantum Design (MPMS-XL).

\section{Polarized neutron reflectivity}

Polarized neutron reflectivity (PNR) measurements for the samples were performed at the reflectometer AMOR at SINQ, Paul Scherrer Institute in Switzerland. ${ }^{\mathbf{1 1}}$ The samples were measured using the time-of flight (TOF) mode. A resolution of $2 \mathrm{~mm}$ was obtained using a position sensitive detector (PSD) positioned about $3 \mathrm{~m}$ behind the sample to detect the neutrons. An in-plane magnetic field of $1 \mathrm{~T}$ was used to saturate the FM layer before the samples were cooled in a closed-cycle cryostat measured at $50 \mathrm{~K}$ and $400 \mathrm{~K}$.

From the neutron polarization analysis we resolved the different components of the magnetization within the film plane as only the magnetic moment within the sample plane contributes to the scattering. The scattering length densities (SLD) of a specimen are given by the nuclear $\left(\rho_{\mathrm{n}}\right)$ and magnetic $\left(\rho_{\mathrm{m}}\right)$ components of the SLD. Two different cross sections were measured, namely the non-spin flip (NSF) channels represented by $R_{+}$(up) and $R_{-}$(down). Here the + and - signs are used to distinguish the intensity contributions $R$ representing the polarization components parallel or anti-parallel to the guiding field, respectively. The NSF scattering amplitude provides information about $\rho_{\mathrm{n}} \pm \rho_{\mathrm{m}} \cos \phi_{\mathrm{A}}$. We designate $\phi_{\mathrm{A}}$ as the angle between the direction of FM magnetization $\left(\mathbf{M}_{\mathrm{FM}}\right)$ and the neutron spin quantization axis. The neutron polarization vector is guided by the field applied to the sample $\left(\mathbf{H}_{\mathrm{a}}\right)$ along the $y$-axis. In the present case, since we measure at a saturation field, $\phi_{\mathrm{A}}=0 . .^{12-14}$

\section{Results and discussion}

$\mathrm{X}$-ray reflectivity (XRR), X-ray diffraction (XRD) and secondary ion mass spectroscopy (SIMS)

Fig. 1(a) shows the XRR data (scanned along $Q_{z}$ ) for the samples grown on $\mathrm{Al}_{2} \mathrm{O}_{3}$ (0001). The reflectivity spectra was analysed by means of a standard fitting routine based on the Parratt algorithm. ${ }^{15}$ The film was modelled as consisting of individual layers of specific thickness, roughness and scattering length density. The simulation reveals that the thickness of the Fe-Rh layer is around $70 \mathrm{~nm}$ with a scattering length density (SLD) value of $7.21 \times 10^{-3} \mathrm{~nm}^{-2}$. In addition, it reveals a different surface layer of $5 \pm 0.5 \mathrm{~nm}$ thickness with a smaller SLD value of $6.17 \times 10^{-3} \mathrm{~nm}^{-2}$, compared to the layer. This SLD value corresponds very well to that of bulk Fe which is a strong indication of the fact that Fe is separated from the FeRh phase during annealing and is accumulated on the top of the film. The substrate SLD value of $2.65 \times 10^{-3} \mathrm{~nm}^{-2}$ was found to be similar to that of amorphous alumina $\left(2.52 \times 10^{-3} \mathrm{~nm}^{-2}\right)$. Low temperature $\left(30{ }^{\circ} \mathrm{C}\right)$ exposure could often lead to amorphous oxide films of a few nanometers.

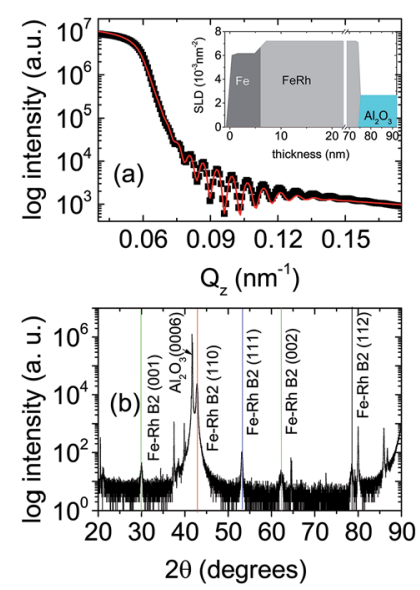

Fig. 1 XRR and XRD data. (a) XRR ( $\left.\mathrm{Cu}-\mathrm{K}_{\alpha}\right)$ patterns of the sample and its fit (red line) are plotted versus $Q_{z}$ at room temperature. Electron density depth profile is shown in the inset. (b) XRD patterns of the polycrystalline sample. 
Room temperature XRD measurement has been performed to probe the crystallinity of the films. High angle XRD data for the sample are shown in Fig. 1(b). One can see the B2-order (001), (110), (111) and (112) reflection peaks. The out-of-plane lattice constant is calculated as $0.2993 \mathrm{~nm}$ from the $(00 l)$ peaks. Thus it is fairly close to the bulk value of $0.2989 \mathrm{~nm} .^{6}$ The grain size obtained from the B2 (110) peak is around $68 \mathrm{~nm}$.

The compositional variation from the surface to the substrate was additionally verified using SIMS. The SIMS intensity ratio between the signals of ${ }^{56} \mathrm{Fe}$ and ${ }^{103} \mathrm{Rh}$ (not shown) confirmed a decay until a depth of $5 \mathrm{~nm}$ which then remains constant before it decays again beyond a depth of $40 \mathrm{~nm}$. This variation is in accordance with our compositional estimates at the surface and bottom interfaces.

\section{Magnetization}

So far the focus of studies has been based upon epitaxial films only due to the structural constraint imposed. Structural constraint guides the physical properties such as metamagnetic transition. Polycrsystallinity could influence the magnetic characteristics significantly. Thus we have compared our polycrystalline film properties with the properties of similarly grown epitaxial films reported in the literature.

In order to characterize the magnetic properties of the polycrystalline sample, the magnetization (M) was measured as a function of temperature at different applied magnetic fields. For epitaxially grown samples it was reported that the films were completely ferromagnetic at $400 \mathrm{~K}$. From this maximum temperature, a temperature sweep was done down to $5 \mathrm{~K}$ and back up to $400 \mathrm{~K}$ again in different magnetic fields from $\mathbf{H}_{\mathrm{a}}=7$ $\mathrm{T}$ to $1 \mathrm{~T}$ in steps of $1 \mathrm{~T}$. The $\mathbf{M}(T)$ curves are shown in Fig. 2(a and b) for cooling and heating of the sample. A remarkable property of the polycrystalline FeRh film is that it does not become completely $\mathrm{AF}$ at low temperatures, but retains a constant magnetization far below $T_{\mathrm{AF}-\mathrm{FM}}$ or $T_{\mathrm{FM}-\mathrm{AF}}$. This constant low magnetization can be clearly observed in the magnetization curves for both the cooling and heating procedures. This was not seen in epitaxially grown FeRh films on $\mathrm{Al}_{2} \mathrm{O}_{3} \cdot{ }^{16}$ However, in an epitaxial film grown on $\mathrm{MgO}$ (001), an FM moment at $80 \mathrm{~K}$ was reported which was attributed to a Rh rich layer at the top with a reduced magnetic moment than that within the film. ${ }^{6}$

In order to determine the nucleation kinetics we plot the change in magnetization upon temperature cycling and field cycling. In epitaxial films, sharpness in the peak, usually seen during cooling, means a homogeneous transition from the FM to AF phase. Similarly, broadness in the peak, usually during heating, signifies a heterogeneous transition from the AF to FM phase. Fig. 2(c and d) show the data for temperature cycling in fields of $1 \mathrm{~T}$ and $7 \mathrm{~T}$. During cooling, epitaxially grown samples had a faster AF nucleation, with an FWHM of only $5 \mathrm{~K}(1 \mathrm{~T}),{ }^{16}$ as compared to $37 \mathrm{~K}(1 \mathrm{~T})$ and $40 \mathrm{~K}(7 \mathrm{~T})$ in our polycrystalline sample. The scenario is quite similar during heating as an FWHM of $20 \mathrm{~K}(1 \mathrm{~T})$ was reported for epitaxial films which is around the $22 \mathrm{~K}(1 \mathrm{~T})$ and $30 \mathrm{~K}(7 \mathrm{~T})$ in our polycrystalline film. The formation of FM domains is assisted by a strong external magnetic field during heating in both the polycrystalline and
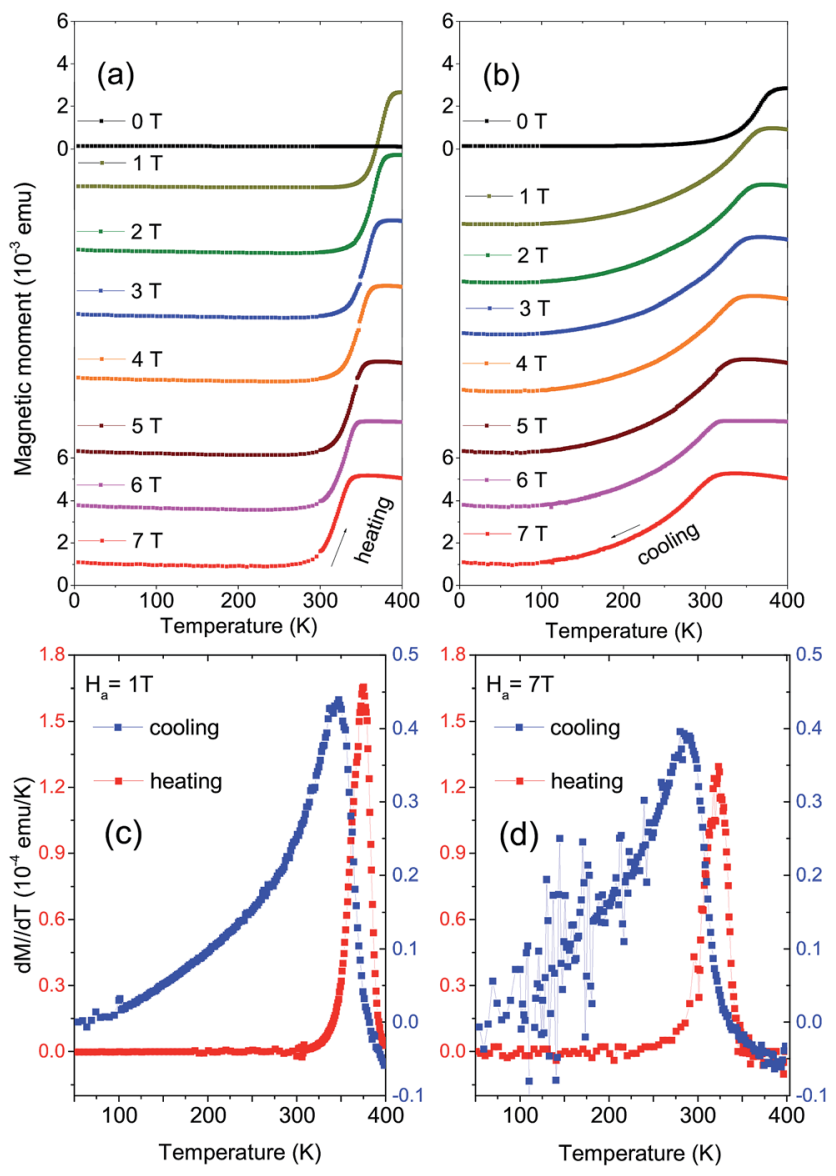

Fig. 2 Magnetization versus temperature measurements and domain nucleation and propagation measurements $d M / d T$. The temperature hysteresis loop measurements at various applied fields for (a) heating and (b) cooling. Rate (dM/dT) of FM (during heating) and AF domain nucleation and propagation (during cooling) at (c) $\mathrm{H}_{\mathrm{a}}=1 \mathrm{~T}$ and (d) $7 \mathrm{~T}$. The FM nucleation peak during heating is sharp while the AF nucleation peak during cooling is broad in both fields.

epitaxial films. The broadness of the peak during cooling can be ascribed to the polycrystalline structure of our film. The impurities and grain boundaries inside the polycrystalline film lead to stronger pinning of the domain walls during the nucleation of the AF phase during cooling than in epitaxial ones. We found a reduction of $T_{\mathrm{FM}-\mathrm{AF}}$ from $346 \mathrm{~K}$ in $1 \mathrm{~T}$ to $288 \mathrm{~K}$ in $7 \mathrm{~T}$ during cooling and a reduction of $T_{\mathrm{AF}-\mathrm{FM}}$ from $374 \mathrm{~K}$ in $1 \mathrm{~T}$ to $325 \mathrm{~K}$ in 7 T during heating.

The corresponding temperature versus field phase diagrams ( $T-H$ diagrams) are shown in Fig. 3(c and d). The values for $T_{\mathrm{AF}-}$ FM and $T_{\mathrm{FM}-\mathrm{AF}}$ are obtained from the temperature hysteresis loop measurements in fixed fields. The phase diagram plots were fitted with a slope of $\mathrm{d} T / \mathrm{d} H=-9.6 \mathrm{~K} \mathrm{~T}^{-1}$ (during cooling) and $-8.5 \mathrm{~K} \mathrm{~T}^{-1}$ (during heating). According to McKinnon et al. ${ }^{17}$ the AF-FM phase transition can be described by an empirical expression

$$
\frac{H}{H_{0}}=1-\left(\frac{T}{T_{0}}\right)^{2}
$$



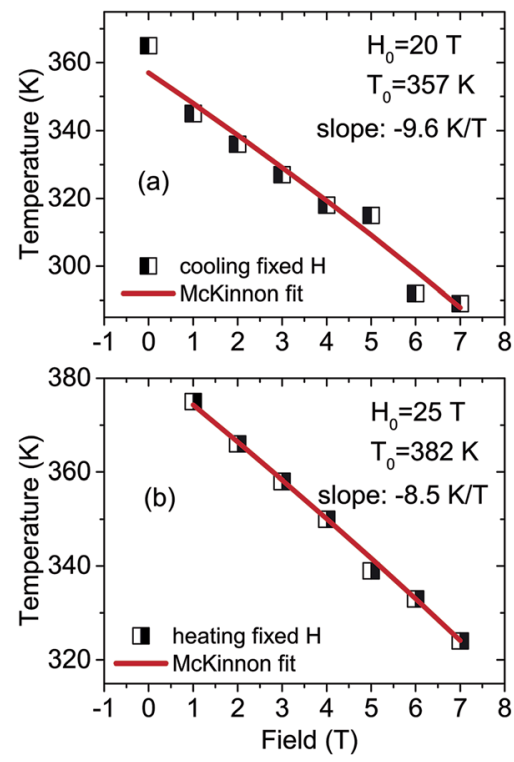

Fig. $3 \mathrm{~T}-\mathrm{H}$ phase diagram as determined from the temperature hysteresis loops in fixed magnetic fields for (a) cooling and (b) heating.

where $H_{0}$ and $T_{0}$ describe the transition field at $T=0 \mathrm{~K}$ and transition temperature at $H=0 \mathrm{~T}$, respectively. Thus from the fits we obtain, $H_{0}=20 \mathrm{~T}$ (during cooling) with $T_{0}=357 \mathrm{~K}$ and $H_{0}=25 \mathrm{~T}$ (during heating) with $T_{0}=382 \mathrm{~K}$.

In-plane magnetic field hysteresis loops were measured at different temperatures for the cooling and heating cycles and are shown in Fig. 4(a and d). The measurements were done on cooling (heating) from $400 \mathrm{~K}$ (from $100 \mathrm{~K}$ ) in $\mathbf{H}_{\mathrm{a}}=0 \mathrm{~T}$. Before each measurement, we demagnetized the sample at a measuring temperature and then measured while sweeping the magnetic field from $0 \mathrm{~T}$ to $+7 \mathrm{~T}$ and finally completing a cycle between $+7 \mathrm{~T}$ to $-7 \mathrm{~T}$. One can see that, unlike epitaxial films on $\mathrm{Al}_{2} \mathrm{O}_{3},{ }^{16}$ the sample is not in the $\mathrm{AF}$ phase at zero field during the cooling process. Spontaneous magnetization develops with field reduction. However, during the heating process, it remains in the AF phase at $\mathbf{H}_{\mathrm{a}}=0 \mathrm{~T}$. The rate of FM and $\mathrm{AF}$ nucleation and propagation can be seen in the plots Fig. 4(b, c, e and f) during cooling and heating, respectively. A similar FWHM of $0.02 \mathrm{~T}$ is observed in all cases, indicating similar transition rates from the FM-to-AF and AF-to-FM phases. Another notable aspect is the order of magnitude reduction in the saturation fields $(0.01 \mathrm{~T})$ as compared to epitaxial ones on $\mathrm{Al}_{2} \mathrm{O}_{3}$.

\section{Polarized neutron reflectivity (PNR)}

Fig. 5(a and b) shows the polarized neutron intensity profiles along $Q_{z}$ and their fits after cooling the sample from $400 \mathrm{~K}$ in a field of $\mathbf{H}_{\mathrm{a}}=1 \mathrm{~T}$ and measuring at $1 \mathrm{~T}$ at $400 \mathrm{~K}$ and $50 \mathrm{~K}$. The sample is expected to be ferromagnetic at $400 \mathrm{~K}$ and antiferromagnetic at $50 \mathrm{~K}$. The fits were done using a simple model of block-potentials. The parameters that were used for fitting are the individual layer thicknesses, and the nuclear and magnetic SLDs of the individual layers. The error in the thickness of the layers is $\pm 0.2 \mathrm{~nm}$, while that for the nuclear and magnetic
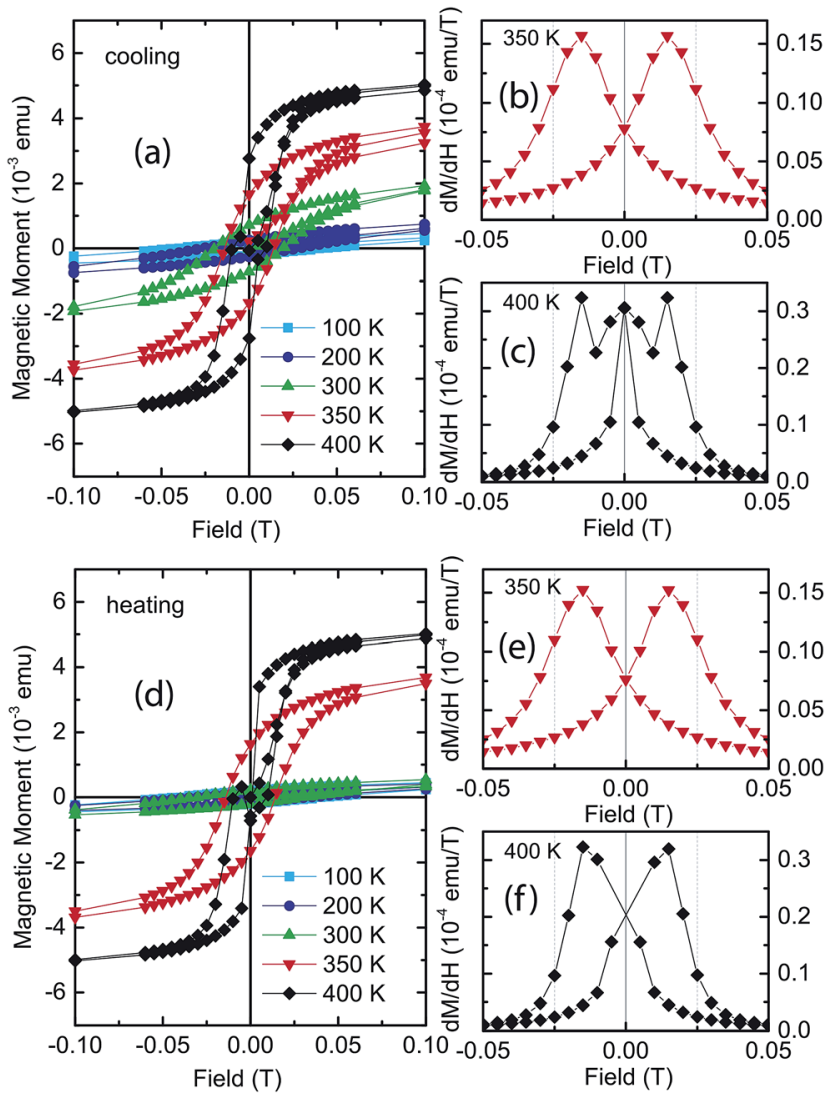

Fig. 4 Magnetization versus field measurements and domain nucleation and propagation $\mathrm{d} M / \mathrm{d} H$. Field hysteresis loops measured at 100 $\mathrm{K}, 200 \mathrm{~K}, 300 \mathrm{~K}, 350 \mathrm{~K}$ and $400 \mathrm{~K}$ during (a) cooling and (d) heating procedures. Rate of $\mathrm{FM}$ and $\mathrm{AF}$ domain nucleation and propagation for ( $b$ and $c$ ) cooling and (e and f) for heating at two different temperatures $T=350 \mathrm{~K}$ and $400 \mathrm{~K}$.

scattering length densities $\rho_{\mathrm{n}}$ and $\rho_{\mathrm{m}}$ values are $\pm 0.1 \times 10^{-6} \AA^{-2}$ and $\pm 0.05 \times 10^{-6} \AA^{-2}$, respectively. The interface roughness is $\simeq 0.5 \pm 0.5 \mathrm{~nm}$. The magnetic scattering length densities of the top-interface and substrate-interface layers were fitted independently from the rest. The substrate $\rho_{\mathrm{n}}$ value at $4.5 \times$ $10^{-6} \AA^{-2}$, was found to be close to the theoretical SLD value of amorphous alumina $\left(4.3 \times 10^{-6} \AA^{-2}\right)$ and in agreement with our X-ray SLD value.

\section{PNR data at $400 \mathrm{~K}$}

The PNR data measured at $400 \mathrm{~K}$ (Fig. 5(a)) show a large splitting of the $R_{+}$and $R_{-}$profiles, a signature of net magnetization within the sample. The nuclear SLD $\rho_{\mathrm{n}}=5.5 \times 10^{-6} \AA^{-2}$ is similar to the expected value $\left(\rho_{\mathrm{n}}=5.7 \times 10^{-6} \AA^{-2}\right)$ in epitaxial films. ${ }^{6}$ Interestingly, the SLD values at the bottom and at the top interfaces are distinctly different in our film. Note that the $\rho_{\mathrm{n}}$ value at the top-interface $\left(\rho_{\mathrm{n}(\mathrm{top})}=7.4 \times 10^{-6} \AA^{-2}\right)$ is reasonably close to the theoretical value $\left(\rho_{\mathrm{n}(\mathrm{Fe})}=8.0 \times 10^{-6} \AA^{-2}\right)$ for Fe, which strongly suggests Fe segregation which is extended to within $10 \mathrm{~nm}$ from the top. The substrate-interface, within $2.5 \mathrm{~nm}$ from the bottom, has $\rho_{\mathrm{n} \text { (bottom) }}=3.8 \times 10^{-6} \AA^{-2}$, 


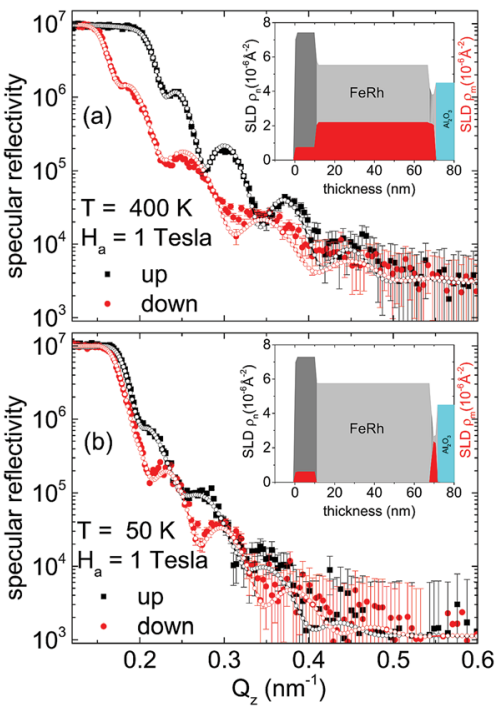

Fig. 5 PNR measurements. Specular neutron reflectivity patterns (solid symbols) along with their best fits (open symbols) as a function of $Q_{z}$ for the NSF $\left[R_{+}\right.$(black) and $R_{-}$(red)] channels, up and down, measured at $\mathrm{H}_{\mathrm{a}}=1 \mathrm{~T}$ and at (a) $400 \mathrm{~K}$ and (b) $50 \mathrm{~K}$. The nuclear $\left(\rho_{\mathrm{n}}\right)$ and magnetic $\left(\rho_{\mathrm{m}}\right)$ SLDs versus the thickness of the sample are also shown inset.

suggesting a $\mathrm{Rh}$ rich interface layer which is similar to the theoretical value for $\mathrm{Rh}\left(\rho_{\mathrm{n}(\mathrm{Rh})}=4.3 \times 10^{-6} \AA^{-2}\right)$.

The magnetic SLD within the film is $\rho_{\mathrm{m}}=2.22 \times 10^{-6} \AA^{-2}$, which is equivalent to $1.11 \mu_{\mathrm{B}}$ per atom of FeRh. The magnetic moment within the polycrystalline film is much lower as compared to that in an epitaxial film (1.56 $\mu_{\mathrm{B}}$ per atom of FeRh) and also to that in the bulk (2.0 $\mu_{\mathrm{B}}$ per atom of FeRh). ${ }^{6}$ Overall, the smaller magnetic moment in our polycrystalline film may be due to a slight imbalance in the 50-50 FeRh composition with a richness of $\mathrm{Rh}$.

Within $10 \mathrm{~nm}$ from the top-interface, $\rho_{\mathrm{m}(\mathrm{top})}=0.77 \times 10^{-6} \AA^{-2}$, which is drastically reduced $\left(\approx 0.39 \mu_{\mathrm{B}}\right.$ per atom of FeRh) from the rest of the film. The reduction of moment at the top is also drastic as compared to that reported in the epitaxial film $\left(\approx 1.32 \mu_{\mathrm{B}}\right.$ per atom of FeRh). ${ }^{6}$ Within around $2.5 \mathrm{~nm}$ of the substrate-interface at the bottom, we find $\rho_{\mathrm{m} \text { (bottom) }}=2.13 \times$ $10^{-6} \AA^{-2}\left(\approx 1.07 \mu_{\text {B }}\right.$ per atom of FeRh). However, the moment value near the substrate-interface in the polycrystalline film is higher than the value reported $\left(0.08 \mu_{\mathrm{B}}\right.$ per atom of FeRh) in the epitaxial film. Thus we can infer that at the substrate-interface, we can have a Rh enrichment which is higher than within the film.

\section{PNR data at $50 \mathrm{~K}$}

The PNR data measured at $50 \mathrm{~K}$ in Fig. 5(b) show a small splitting of the $R_{++}$and $R_{--}$profiles, a signature of reduced net magnetization within the sample. The magnetic moment at the substrate-interface $\left(\approx 1.18 \mu_{\mathrm{B}}\right.$ per atom of FeRh), and at the topinterface remain similar $\left(0.31 \mu_{\mathrm{B}}\right.$ per atom of FeRh) to that at $400 \mathrm{~K}$. The inner FeRh layer is completely antiferromagnetic. One may recall the non-vanishing magnetic moment below
$T_{\mathrm{FM}-\mathrm{AF}}$, as observed in the SQUID data (Fig. 2(a and b)). The persistent moment at $50 \mathrm{~K}$ can therefore be explained as being mostly reminiscent of the Fe rich top-interface and $\mathrm{Rh}$ rich substrate-interface layers at $400 \mathrm{~K}$. The temperature dependence of the magnetization can be related to the magnetic phase diagram of FeRh alloys.

\section{Model simulations}

In order to justify the variations in $\rho_{\mathrm{m}}$ with thickness, we present model simulations considering (a) a higher $\rho_{\mathrm{m}}$ value at the top-interface as compared to the rest of the layer and (b) a constant average value of $\rho_{\mathrm{m}}=1 \times 10^{-6} \AA^{-2}$ for the data at 400 K. The simulated data are shown in Fig. 6(a and b). It is quite obvious that such scenarios can be ruled out. Similarly, for the $50 \mathrm{~K}$ data, we again present model simulations considering (a) a higher $\rho_{\mathrm{m}}$ value at the top-interface with no magnetization for the rest of the layer and (b) a constant average value of $\rho_{\mathrm{m}}=0.6$ $\times 10^{-6} \AA^{-2}$. The simulated data are shown in Fig. 6(c and $d$ ) confirming that such models fail to match the data.

\section{Disordered phases}

Fig. 7 shows the phase diagram of bulk $\mathrm{Fe}_{x} \mathrm{Rh}_{100-x}$ alloys. ${ }^{18}$ It is well known that in the range of $0-20$ atomic percent (at\%) of $\mathrm{Rh}$, the alloy is in a chemically disordered bcc structure (A2). Thus the $\mathrm{Rh}$ rich bottom-interface shows some low magnetism. The long-range magnetic order of A2-FeRh arises from the dominating short-range FM exchange interactions between $\mathrm{Fe}-\mathrm{Fe}$ and $\mathrm{Fe}-\mathrm{Rh}$ pairs over the competing $\mathrm{AF}$ Fe-Fe interactions at larger separations..$^{19,20}$

When the system is in between 20 and 50 at\% of Rh, it goes into a chemically ordered bcc structure (B2). Then with $x<50$, i.e. when $\mathrm{Rh}$ is more than around 50 at\%, it has a fcc structure and is not magnetic. Using Mössbauer spectroscopy, Filoti et al. ${ }^{21}$ studied magnetism in $\mathrm{Fe}_{x} \mathrm{Rh}_{100-x}$ bulk alloys at different temperatures, where $x=65,50$ and 26. A transition from the paramagnetic (PM) to FM phase between $375-525 \mathrm{~K}$ for $x=65$ was demonstrated from the evolution of a sextet. It was shown that the bcc disordered phase A2 contains about $16 \%$ of the paramagnetic phase. Therefore, the loss of magnetization at the top-interface as compared to the inner portion of our sample, which is associated with Fe segregation, is caused by a disordered bcc FM phase or a near-surface/interfacial ferromagnetism in the ordered FeRh thin films.

\section{Summary}

A polycrystalline FeRh film on $\mathrm{Al}_{2} \mathrm{O}_{3}$ (0001) with predominant B2-order has been investigated. We found $T_{\mathrm{AF}-\mathrm{FM}}=346 \mathrm{~K}$ (in 1 $\mathrm{T}$ ), which can be shifted by a magnetic field to $288 \mathrm{~K}$ (in $7 \mathrm{~T}$ ). The rates of transitions with field $\left(\mathrm{d} T / \mathrm{d} H=-8.5 \mathrm{~K} \mathrm{~T}^{-1}\right.$ for heating and $\mathrm{d} T / \mathrm{d} H=-9.6 \mathrm{~K} \mathrm{~T}^{-1}$ for cooling) are similar to those in epitaxial films. We find a significant difference in the rate of domain nucleation and propagation with temperature $(\mathrm{d} M / \mathrm{d} T)$ during the cooling and heating procedures. Using PNR, we show that the FeRh film is not homogeneous throughout the depth of the film. Evidence of Fe segregation at the surface of 

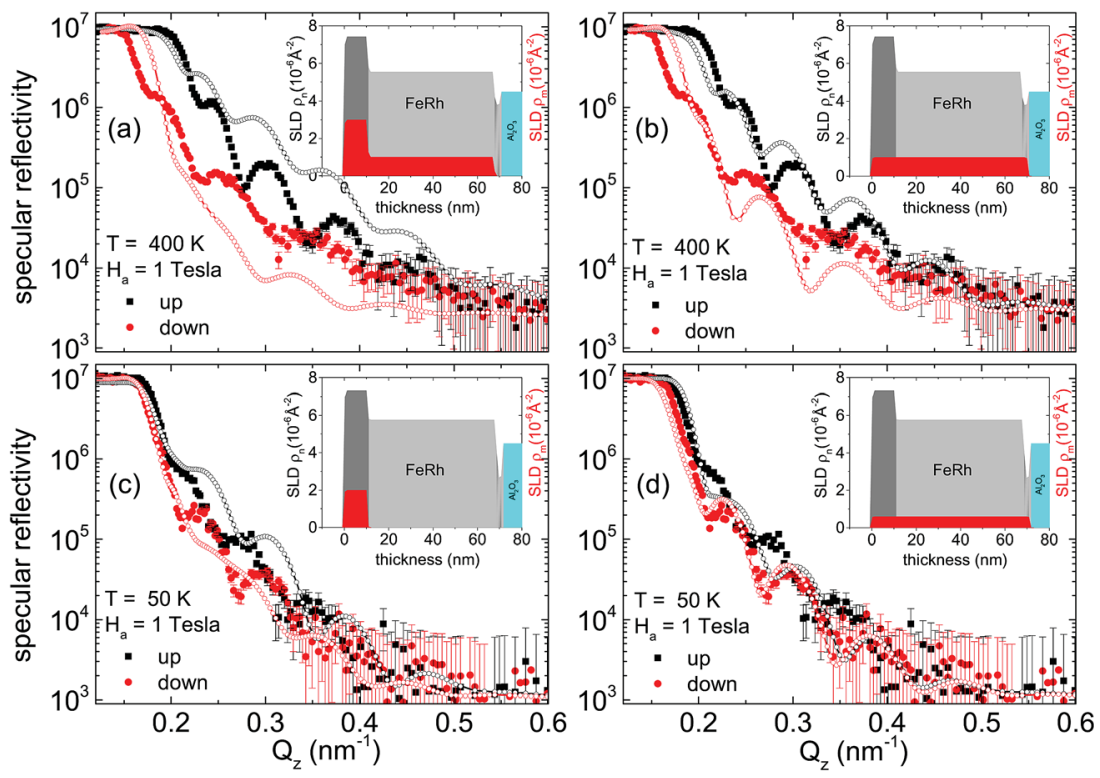

Fig. 6 PNR measurements. Specular neutron reflectivity patterns (solid symbols) along with model simulations (open symbols) as a function of $Q_{z}$ for the NSF $\left[R_{+}\right.$(black) and $R_{-}$(red)] channels, up and down, measured at $\mathrm{H}_{\mathrm{a}}=1 \mathrm{~T}$ and at (a and b) $400 \mathrm{~K}$ and (c and d) $50 \mathrm{~K}$. The nuclear ( $\rho_{\mathrm{n}}$ ) and magnetic $\left(\rho_{\mathrm{m}}\right)$ SLDs versus the thickness of the sample are also shown inset. We also show the model simulations considering two different models each for $400 \mathrm{~K}$ and $50 \mathrm{~K}$ with different $\rho_{\mathrm{m}}$ values. The mismatch with the data indicates the invalidity of the model in each case.

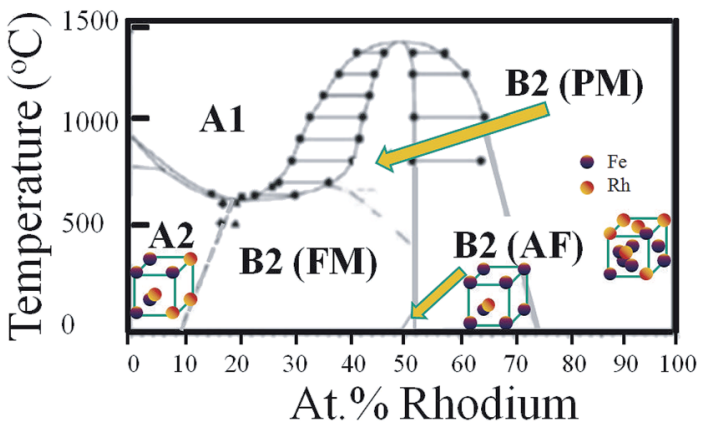

Fig. 7 FeRh phase diagram. Bulk magnetic $\mathrm{Fe}_{x} \mathrm{Rh}_{100-x}$ phase diagram adapted from ref. 18. Metamagnetic transitions in bulk B2 FeRh are expected from the AF to the FM state at $370 \mathrm{~K}$ and to the PM state at $655 \mathrm{~K}$. The green arrows indicate the 50-50 B2 ordered phase, being magnetic and paramagnetic. Below $10 \%$ of $\mathrm{Rh}$, it is in a disordered bcc phase (A2) and above $75 \%$ of Rh it is in a disordered fcc (A1) phase. Schematic diagrams of the crystal structures for $A 2, B 2$ and $A 1$ are also shown.

the film extended to within a few nanometers from the topinterface and $\mathrm{Rh}$ richness at the substrate-interface was confirmed. Overall, differences to the epitaxial film can be ascribed to the ordered and disordered polycrystallinity of the film. The inhomogeneous film changes from a disordered bcc (A2) structure at the surface to an ordered bcc (B2) structure within the film and then to a disordered fcc phase (A1) at the very bottom. We expect that, with improved growth conditions, it would be possible to grow simpler and cost effective polycrystalline FeRh films possessing similar characteristics as epitaxial ones.

\section{Conflicts of interest}

The authors declare no competing financial interests.

\section{Acknowledgements}

V. S., R. R., M. G., S. A. and A. T. prepared the sample. J. Y. and M. H. did the magnetization measurements. R. R., M. G. and J. S. did the PNR measurements. A. P. analyzed the data, coordinated the work, and wrote the manuscript. We would like to thank M. Opel for some of the SQUID measurements and N. Paul for the X-ray measurements. We are also thankful to E. Hueger for the SIMS measurements. The work is partially based upon the experiments performed on AMOR at the Swiss spallation neutron source SINQ, Paul Scherrer Institute in Switzerland. This work was supported by the German Research Foundation (DFG) and the Technische Universität München within the Open Access Publishing Funding Programme.

\section{References}

1 M. Fallot and R. Horcart, Rev. Sci., 1939, 77, 498.

2 F. de Bergevin and L. Muldawer, Comptes Rendus de l'Académie des Sciences, 1961, 252, 1347.

3 P. Kushwaha, A. Lakhani, R. Rawat and P. Chaddah, Phys. Rev. B: Condens. Matter Mater. Phys., 2009, 80, 174413.

4 N. Fujita, S. Kosugi, Y. Saitoh, Y. Kaneta, K. Kume, T. Batchuluun, N. Ishikawa, T. Matsui and A. Iwase, J. Appl. Phys., 2010, 107, 09E302.

5 K. Kang, A. R. Moodenbaugh and L. H. Lewis, Appl. Phys. Lett., 2007, 90, 153112. 
6 R. Fan, C. J. Kinane, T. R. Charlton, R. Dorner, M. Ali, M. A. de Vries, R. M. D. Brydson, C. H. Marrows, B. J. Hickey, D. A. Arena, B. K. Tanner, G. Nisbet and S. Langridge, Phys. Rev. B: Condens. Matter Mater. Phys, 2010, 82, 184418.

7 R. O. Cherifi, V. Ivanovskaya, L. C. Phillips, A. Zobelli, I. C. Infante, E. Jacquet, V. Garcia, S. Fusil, P. R. Briddon, N. Guiblin, A. Mougin, A. A. Unal, F. Kronast, S. Valencia, B. Dkhil, A. Barthelemy and M. Bibes, Nat. Mater., 2014, 13, 345 .

8 S. Yamada, K. Tanikawa, J. Hirayama, T. Kanashima, T. Taniyama and K. Hamaya, Phys. Rev. B: Condens. Matter Mater. Phys., 2015, 92, 094416.

9 M. Takahashi and R. Oshima, J. Phys. IV, 1995, 05, C8-C491.

10 R. Witte, R. Kruk, M. E. Gruner, R. A. Brand, D. Wang, S. Schlabach, A. Beck, V. Provenzano, R. Pentcheva, H. Wende and H. Hahn, Phys. Rev. B, 2016, 93, 104416.

11 J. Stahn and A. Glavic, Nucl. Instrum. Methods Phys. Res., Sect. $A, 2016,821,44$.
12 J. Jutimoosik, R. Yimnirun, J. Stahn, A. Setzer, P. Esquinazi and A. Paul, Phys. Rev. B: Condens. Matter Mater. Phys., 2015, 91, 224428.

13 S. Fust, S. Mukherjee, N. Paul, J. Stahn, W. Kreuzpaintner, P. Böni and A. Paul, Sci. Rep., 2016, 6, 33986.

14 A. Paul, Sci. Rep., 2016, 6, 19315.

15 L. G. Paratt, Phys. Rev., 1954, 95, 359.

16 S. Maat, J.-U. Thiele and E. E. Fullerton, Phys. Rev. B: Condens. Matter Mater. Phys., 2005, 72, 214432.

17 J. B. McKinnon, D. Melville and E. W. Lee, J. Phys. C: Solid State Phys., 1970, 1, S46.

18 V. Dupuis, A. Robert, A. Hillion, G. Khadra, N. Blanc, D. L. Roy, F. Tournus, C. Albin, O. Boisron and A. Tamion, Beilstein J. Nanotechnol., 2016, 7, 1850.

19 J. Kudrnovský, V. Drchal and I. Turek, Phys. Rev. B: Condens. Matter Mater. Phys., 2015, 91, 014435.

20 I. Ohnuma, T. Gendo, R. Kainuma, G. Inden and K. Ishida, ISIJ Int., 2009, 49, 1212.

21 G. Filoti, V. Kuncsea, E. Navarro, A. Hernando and M. Rosenberg, J. Alloys Compd., 1998, 278, 60. 